\title{
First-principles calculation of the effects of partial alloy disorder on the static and dynamic magnetic properties of $\mathrm{Co}_{2} \mathrm{MnSi}$
}

\author{
B. Pradines, ${ }^{*}$ R. Arras, I. Abdallah, N. Biziere, and L. Calmels \\ CEMES, CNRS, Université de Toulouse, 29 rue Jeanne Marvig, F-31055, Toulouse, France \\ (Received 27 October 2016; revised manuscript received 20 January 2017; published 22 March 2017)
}

\begin{abstract}
On the basis of fully relativistic Korringa-Kohn-Rostoker calculations and in conjunction with the coherent potential approximation and the linear response formalism, we present a complete $a b$ initio study of the influence of alloy disorder on the static and dynamic (Gilbert damping) magnetic properties and on the electronic structure of the half-metallic full-Heusler alloy $\mathrm{Co}_{2} \mathrm{MnSi}$. We discuss in particular partial atomic disorders intermediate between the main crystal phases $\mathrm{L} 2{ }_{1}, \mathrm{~B} 2, \mathrm{~A} 2$, and $\mathrm{D} 0_{3}$ of this alloy. We compare our results with homemade experiments and measurements from the literature, and conclude that the presence of a partial $\mathrm{D}_{3}$-like disorder could explain the relatively high value of the Gilbert damping parameter and the lack of half-metallicity measured in real samples, in which alloy disorder cannot be totally avoided.
\end{abstract}

DOI: 10.1103/PhysRevB.95.094425

\section{INTRODUCTION}

The last decades have seen a growing interest for magnetic materials, allowing the conception of new spintronic devices. The key features of an ideal magnetic material for spintronic applications are the following: a high Curie temperature in order to enable devices to work at room temperature, a high spin-polarization at the Fermi level $P\left(E_{F}\right)$ in order to get high tunnel magnetoresistance (TMR) effects, and a low damping parameter of the magnetic relaxation (as described in the Landau-Lifshitz-Gilbert equation [1]) to allow an easy switching of the magnetization by spin-transfer torque effect [2].

A magnetic compound family fulfilling all the requirements stood out and attracted the attention of many physicists because of its great magnetic versatility: the full-Heusler alloy family, discovered in 1903 [3], is characterized by the chemical formula $\mathrm{X}_{2} \mathrm{YZ}$ and the crystal space group $F m \overline{3} m$ for its most ordered $\mathrm{L} 2{ }_{1}$ cubic phase, where the atoms $\mathrm{X}$, $\mathrm{Y}$, and $\mathrm{Z}$ occupy respectively the Wyckoff positions 8c, 4a, and $4 \mathrm{~b}$. A tuning of the magnetic properties is possible by adjusting the chemical composition of the alloy, making this family of materials very popular. Heusler compounds are presently studied for numerous purposes such as spin-injector electrodes [4], magnetoresistance device electrodes [5-8], or as spin-torque oscillators [9]. For a most detailed review on the Heusler alloys, see Refs. [10-13].

Belonging to this family of materials, the full-Heusler alloy $\mathrm{Co}_{2} \mathrm{MnSi}$ is a very promising candidate for spintronic applications. Since the theoretical prediction of its half-metallicity by first-principles calculations [14], intensive experimental and numerical studies have been performed on this material, highlighting a low damping parameter [15-25], in addition to the already known high Curie temperature [26].

Nevertheless, the expected $100 \%$ spin polarization of $\mathrm{Co}_{2} \mathrm{MnSi}$ at the Fermi level has not been measured so far. The highest value of the spin-polarization found in the literature is around $93 \%$ at room temperature [27] and giant tunneling magnetoresistance ratios, of about $1400 \%$ at low temperature (300\% at room temperature), have been measured recently in

*barthelemy.pradines@ cemes.fr magnetic tunnel junctions made with the off-stoichiometric Heusler alloy $\mathrm{Co}_{2} \mathrm{MnSi}_{0.84}$, suggesting a very strong spin polarization of the Heusler alloy electrodes [28]. However, most of the earlier works only reported spin-polarizations around 50\%-60\% [5,29-32], even at low temperature. Atomic disorder has been evoked to explain the suppression of halfmetallicity and neutron diffraction experiments performed on $\mathrm{Co}_{2} \mathrm{MnSi}$ sputtered thin films confirmed the existence of chemical disorder in this alloy [30,33].

This disorder was only detected between cobalt and manganese atoms, leading to the $\mathrm{DO}_{3}$ crystal phase in the case of a perfect disorder. Starting from these results, Picozzi et al. [34] studied the influence of point defects on the magnetic properties of $\mathrm{Co}_{2} \mathrm{MnSi}$, using density functional theory (DFT) calculations and a supercell method to describe the atomic structure. They concluded that the defects which are responsible for the appearance of electronic states in the minority spin band gap are those that change the first-neighbor chemical environment, like Co-Mn swaps or Co antisites, other defects like Mn-Si swaps having almost insignificant effects on the half-metallicity. These conclusions support the idea that the partial $\mathrm{DO}_{3}$ disorder, potentially present in $\mathrm{Co}_{2} \mathrm{MnSi}$ samples, is responsible for the destruction of the half-metallicity of this compound. This was further confirmed by Pandey et al. [35].

Concerning the Gilbert damping parameter, low values of the order of $4 \times 10^{-3}$ are generally reported in the literature [15-19], reduced below $10^{-3}$ in the most recent studies [20-23]. These values are however still higher than the predicted ones calculated for the pure L $2_{1}$ phase. The Kamberský breathing Fermi surface (BFS) method [36,37] was used in 2009, to obtain a numerical value of the Gilbert damping parameter for the $\mathrm{L}_{1}$ phase of $\mathrm{Co}_{2} \mathrm{MnSi}$ [24]. In 2015, the torque correlation model (TCM) [38,39] was used within the tight-binding linear muffin-tin orbital (LMTO) method, to establish a correlation between the Gilbert damping parameter, the alloy disorder and the density of states at the Fermi level, for the perfectly ordered $\mathrm{L} 2_{1}$ and the perfectly disordered $\mathrm{A} 2$ and $\mathrm{B} 2$ phases of $\mathrm{Co}_{2} \mathrm{MnSi}$ [25]. However, the $\mathrm{DO}_{3}$ phase was not treated there, despite the fact that previous experimental studies suggested its existence to explain the lack of half-metallicity of this alloy. Furthermore, BFS and TCM cannot take into account from first-principles physical effects 
that are responsible for some of the magnetic relaxation paths: these methods rely on a phenomenological parameters $\tau$ that characterizes the lifetime of the electron states. The Gilbert damping parameter of $\mathrm{Co}_{2} \mathrm{MnSi}$ has been calculated recently by first-principles methods, but only for the L $22_{1}$ phase and at finite temperatures $[40,41]$.

The purpose of this paper is to give a complete $a b$ initio study of the influence of alloy disorder on the static and dynamic magnetic properties and on the electronic structure of $\mathrm{Co}_{2} \mathrm{MnSi}$. This study aims to find out if disorder that may occur from the experimental growth of $\mathrm{Co}_{2} \mathrm{MnSi}$ can theoretically explain the qualitative differences between computed and measured physical properties. We will in particular consider crystal phases intermediate between the $\mathrm{L} 22_{1}, \mathrm{~B} 2, \mathrm{~A} 2$, and $\mathrm{DO}_{3}$ phases, which have not been extensively studied in the literature.

After an explanation of the technical details of our calculations in Sec. II, we will describe, in Sec. III, the ideal properties calculated for the perfectly ordered L $2_{1}$ phase. This first set of data will be used as a reference to investigate, in Sec. IV, the impact of atomic disorder on the energetic stability, the electronic structure and the magnetic properties of $\mathrm{Co}_{2} \mathrm{MnSi}$. Finally, all these results will be correlated and discussed in Sec. V before the conclusion.

\section{CALCULATION DETAILS}

All the electronic structure calculations were performed with the spin-polarized relativistic Korringa-Kohn-Rostoker (SPR-KKR) code $[42,43]$, which is commonly used to study Heusler alloys such as $\mathrm{Co}_{2} \mathrm{MnSi}[28,40,41,44-47]$. This code is based on the KKR-Green's function formalism, which uses the multiple scattering theory to calculate the one-electron Green's function of the crystals [43]. This method was chosen to calculate the electronic structure because it allows to treat random chemical disorder between selected atomic sites via the coherent potential approximation (CPA) [48], without the technical and computational constraints that would be imposed by supercell methods.

Ebert et al. derived, from the linear response formalism (LRF), the following expression for the Gilbert damping parameter $\alpha$ [49], taking inspiration from previous results of Brataas et al. [50]:

$$
\alpha_{\mu \nu}=-\frac{\hbar \gamma}{\pi M_{S}} \operatorname{Trace}\left\langle\frac{\partial \hat{H}}{\partial u_{\mu}} \operatorname{Im} G^{+}\left(E_{F}\right) \frac{\partial \hat{H}}{\partial u_{v}} \operatorname{Im} G^{+}\left(E_{F}\right)\right\rangle_{c},
$$

where $G^{+}\left(E_{F}\right)$ is the retarded single-particle Green's function at the Fermi energy, $\gamma$ is the gyromagnetic ratio, $\partial \hat{H} / \partial u$ is the torque operator, and finally, $\langle\ldots\rangle_{c}$ stands for the configurational average of the disordered system. This expression is tensorial, however for our cubic systems, alpha can be replaced by a scalar parameter without loss of generality. For more precisions on the origin and the use of this expression, see Refs. [49,51]. This method is implemented in the SPR-KKR code and provides a parameter-free technique to extract $\alpha$ from the calculated electronic structure, unlike BSF and TCM methods, which use empirical parameters.
Our calculations were performed within the fully relativistic mode, which ensures the best possible treatment of spin-orbit coupling as origin of the magnetic damping [49]. We used the Vosko, Wilk, and Nusair [52] (VWN) local spin-density approximation (LSDA) for the exchange-correlation potential in the atomic sphere approximation (ASA). After convergence tests, we chose to sample the irreducible wedge of the first Brillouin zone with $3000 \mathbf{k}$ vectors for the SCF cycles and with $50000 \mathbf{k}$ vectors for the calculations of $\alpha$. We also found that the calculated lattice parameter and ground-state energy were sensitive to the selected $l$ cutoff of the KKR formalism, which we increased until we reach energy and lattice parameter convergence. For all further calculations, $l_{\max }$ was set to 4 . In Appendix A, we compare the values of the density of states (DOS) at the Fermi level and Gilbert damping parameter calculated for the $\mathrm{L} 2{ }_{1}$ phase of $\mathrm{Co}_{2} \mathrm{MnSi}$ with and without Lloyd formula. These results show that the improvement due to the Lloyd formula is rather small in our case and does not qualitatively change the physical properties of the Heusler alloy, as long as the $l$ cutoff is big enough, which is the case for our calculations.

We performed a comparison between results calculated for the $\mathrm{L} 2{ }_{1}$ phase with the generalized gradient approximation (GGA) Perdew-Ernzerhof-Burke (PBE) [53] and the VWN functionals (see Table III in Appendix A). Given the small differences between the values of the magnetic moments, DOS at the Fermi level and Gilbert damping parameters calculated with these two functionals, we can reasonably expect that our results are not strongly dependent (at least qualitatively) on the chosen functional, when using the experimental lattice parameter.

To treat the disorder, $\mathrm{Co}_{2} \mathrm{MnSi}$ can be described by the formula

$$
\left[\mathrm{Co}_{2-y-z} \mathrm{Mn}_{z} \mathrm{Si}_{y}\right]_{\mathrm{X}}\left[\mathrm{Mn}_{1-x-z} \mathrm{Co}_{z} \mathrm{Si}_{x}\right]_{\mathrm{Y}}\left[\mathrm{Si}_{1-x-y} \mathrm{Co}_{y} \mathrm{Mn}_{x}\right]_{\mathrm{Z}},
$$

where $\mathrm{X}, \mathrm{Y}$, and $\mathrm{Z}$ correspond to the atomic sites described in Introduction, and $x, y$, and $z$ are the parameters that quantify the disorder and which characterize the amount of $\mathrm{Mn}-\mathrm{Si}$, $\mathrm{Co}-\mathrm{Si}$, and Co-Mn swaps, respectively. In the following and according to the previous chemical formula, the disorder will be characterized by the triplet of parameters $(x, y, z)$.

We focused our calculations on disorders intermediate between the four perfectly ordered or perfectly disordered $\mathrm{Co}_{2} \mathrm{MnSi}$ crystal phases the most reported in the literature: the perfectly ordered L2 2 phase $(0,0,0)$ described in the introduction, the $\mathrm{B} 2$ phase $\left(\frac{1}{2}, 0,0\right)$ for witch $\mathrm{Y}$ and $\mathrm{Z}$ atomic sites are randomly and identically occupied by $\mathrm{Mn}$ and $\mathrm{Si}$ atoms, the $\mathrm{DO}_{3}$ phase $\left(0,0, \frac{2}{3}\right)$ where $\mathrm{Co}$ and $\mathrm{Mn}$ atoms are randomly distributed among $\mathrm{X}$ and $\mathrm{Y}$ atomic sites and the perfectly disordered $\mathrm{A} 2\left(\frac{1}{4}, \frac{1}{2}, \frac{1}{2}\right)$ phase where all atomic sites contain the same amounts of $\mathrm{Co}, \mathrm{Mn}$, and $\mathrm{Si}$ atoms, still with a random distribution. The three variation paths between these four stoichiometric phases that we will describe in Sec. IV are then the following: (a) $(0,0, z)$ with $0 \leqslant z \leqslant \frac{2}{3}$, for disordered phases intermediate between $\mathrm{L}_{1}$ and $\mathrm{D0}_{3}$; (b) $(x, 0,0)$ with $0 \leqslant x \leqslant \frac{1}{2}$, for disordered phases intermediate between $\mathrm{L} 22_{1}$ and $\mathrm{B} 2$; and (c) $\left(x=\frac{1-y}{2}, y, z=y\right)$ with $0 \leqslant$ $y \leqslant \frac{1}{2}$, for disordered phases intermediate between B2 and A2. An additional perfectly disordered phase involving only 
TABLE I. Minority-spin energy band gap, total magnetic moments $\left(M_{\text {spin }}\right)$ per formula unit (f.u.) and for each chemical species, Gilbert damping parameter $\alpha$ and lattice constant $a_{0}$ of the perfectly ordered $\mathrm{L} 2_{1}$ phase of $\mathrm{Co}_{2} \mathrm{MnSi}$, calculated in the present paper and compared to results from the literature. The Gilbert damping parameter reported in the first two lines has been calculated for a nearly L2 1 phase (B2-like disorder with $x=0.01$ ).

\begin{tabular}{|c|c|c|c|c|c|c|c|}
\hline & $a_{0}(\AA)$ & Gap (eV) & $M_{\text {spin }}\left(\mu_{B} /\right.$ f.u. $)$ & $M_{C o}\left(\mu_{B}\right)$ & $M_{M n}\left(\mu_{B}\right)$ & $M_{S i}\left(\mu_{B}\right)$ & $\alpha$ \\
\hline Present paper (KKR, LSDA, LRF) & 5.48 (calc) & 0.48 & 4.88 & 1.02 & 2.88 & -0.05 & $1.6 \times 10^{-4}$ \\
\hline Present paper (KKR, LSDA, LRF) & $5.65(\exp )$ & 0.41 & 4.87 & 0.97 & 3.01 & -0.08 & $1.5 \times 10^{-4}$ \\
\hline KKR, LSDA [14] & $5.65(\exp )$ & 0.35 & 5.00 & 1.00 & 3.05 & -0.06 & - \\
\hline FLAPW, ${ }^{a}$ LSDA [56] & 5.51 (calc) & 0.60 & 5.0 & 1.07 & 2.81 & -0.02 & - \\
\hline FLAPW, GGA ${ }^{\mathrm{b}}[56]$ & 5.64 (calc) & 0.81 & 5.0 & 1.06 & 2.92 & -0.04 & - \\
\hline ASW FSKKR, ${ }^{\mathrm{c}}$ LSDA [57] & $5.65(\exp )$ & 0.63 & 4.94 & 1.02 & 2.97 & -0.07 & - \\
\hline FLAPW, GGA [58] & $5.65(\exp )$ & 0.82 & 5.0 & 1.06 & 2.97 & -0.03 & - \\
\hline PAW, ${ }^{\text {a }}$ EHTB,${ }^{d}$ BSF [24] & $5.65(\exp )$ & - & - & - & - & - & $0.6 \times 10^{-4}$ \\
\hline KKR, tight-binding, TCM [25] & $5.65(\exp )$ & - & - & - & - & - & $1.1 \times 10^{-4}$ \\
\hline
\end{tabular}

${ }^{a}$ Full-potential linearized-augmented plane-wave approximation.

${ }^{\mathrm{b}}$ Generalized gradient approximations.

${ }^{\mathrm{c}}$ Augmented spherical wave full-potential screened KKR approximation.

${ }^{\mathrm{d}}$ Extended Hückel tight-binding approximation.

$\mathrm{Co} / \mathrm{Si}$ swaps and corresponding to the triplet $\left(0, \frac{2}{3}, 0\right)$ will also be briefly discussed to complete our study, but not analyzed in details, as we did not find any mention on its existence in the experimental literature. This phase will be labeled $\mathrm{D}_{3}{ }^{\prime}$.

Calculations are performed at $0 \mathrm{~K}$. However, as the method chosen to calculate the Gilbert damping parameter $\alpha$ from the linear response formalism cannot be used for a perfectly ordered crystal at this temperature, the value of $\alpha$ is never calculated for the perfectly ordered $\mathrm{L} 2{ }_{1}$ phase. The phases the closest from the perfect $\mathrm{L} 2{ }_{1}$ for which $\alpha$ has been calculated possess a very small amount of swapping, either between $\mathrm{Mn}$ and $\mathrm{Si}$ atoms (B2-like disorder with $x=0.01$ ), or between $\mathrm{Co}$ and $\mathrm{Mn}$ atoms $\left(\mathrm{DO}_{3}\right.$-like disorder with $\left.z=0.02\right)$. We checked that a tiny B2-like disorder has nearly no impact on the electronic structure: no significant differences were found between the magnetic and electronic properties of the genuine $\mathrm{L} 2{ }_{1}$ and the nearly ideal L2 $2_{1}$ (B2-like disorder with $x=0.01$ ) crystals. When it will be necessary to compare $\alpha$ with results from the literature for the perfect $\mathrm{L} 2_{1}$ phase, we will thus consider this pseudo-L2 1 phase.

We performed the volume optimization of the four cubic phases $\mathrm{L}_{2}, \mathrm{~B} 2, \mathrm{~A} 2$, and $\mathrm{D}_{3}$ of $\mathrm{Co}_{2} \mathrm{MnSi}$ in order to seek for any change in the lattice parameter resulting from the chemical disorder. The differences between the lattice parameters calculated for the four phases were less than $1 \%$. Experimentally, structural defects (alloy disorder, stoichiometry defects, vacancies, lattice mismatch with a substrate, etc.) can cause small modifications of the crystal structure, in particular of the lattice parameter [41,54,55]. These modifications are nonetheless small and are generally neglected in $a b$ initio calculations [28,44,45]. Consequently, we considered that the chemical disorder does not considerably affect the volume of the cubic cell, for which we used the same lattice constant throughout the paper.

All the results have been calculated with two distinct lattice parameters: the experimental one $a_{0}^{\exp }=5.65 \AA$ [26], and that calculated with the SPR-KKR code after volume optimization, i.e., which corresponds to the minimum value of the total energy, $a_{0}^{\mathrm{DFT}}=5.48 \AA$. Although most of the figures show data calculated both for $a_{0}^{\mathrm{DFT}}$ and for $a_{0}^{\mathrm{exp}}$, we only discuss in details, throughout the paper, the results which have been obtained for $a_{0}^{\exp }$. The comparison between the physical properties calculated for the two lattice parameters is only given in Sec. IV E.

\section{PROPERTIES OF THE PERFECTLY ORDERED L2 1 PHASE}

In this section, we describe the ideal properties of the perfectly ordered $\mathrm{L} 2{ }_{1}$ phase of $\mathrm{Co}_{2} \mathrm{MnSi}$. Our results, compared in Table I to data found in the literature, will be used as references in the next sections, in order to understand the impact of the atomic disorder.

In Fig. 1(a), we plotted the density of states (DOS) computed using the measured value of the lattice constant. Half-metallicity is confirmed, with a nonzero DOS at the Fermi level $\left(E_{F}\right)$ for the majority spin electrons and a band gap for minority spin electrons, as already calculated in previous studies [14,56,57]. The band-gap width that we calculated is between the LSDA values reported in Ref. [14] and Ref. [57] for the same lattice parameter, and almost twice smaller than the values obtained with the generalized gradient approximation (GGA) [56,58]. Figure 1(a) shows that the Fermi energy is located near the center of the minority-spin band gap.

Co atoms having the highest number of electrons, with more than half-filled $d$ orbitals, they bring the main contribution to the minority spin density of occupied states. The Fermi level is located in the minority-spin band gap, between the occupied $d$ bands of Co atoms and states resulting from an hybridization of Co and Mn $d$ orbitals. This has for consequence that the chemical environment around Co atoms will be determinant to keep the half-metallicity.

The ferromagnetic phase is found to be the ground state, with a total magnetic moment $M_{\text {tot }}$ of $4.94 \mu_{B}$ per formula unit (f.u.), in agreement with the values reported in previous numerical investigations (we only found an average difference of $2 \%$ on the total spin magnetic moment). Mn is the chemical 

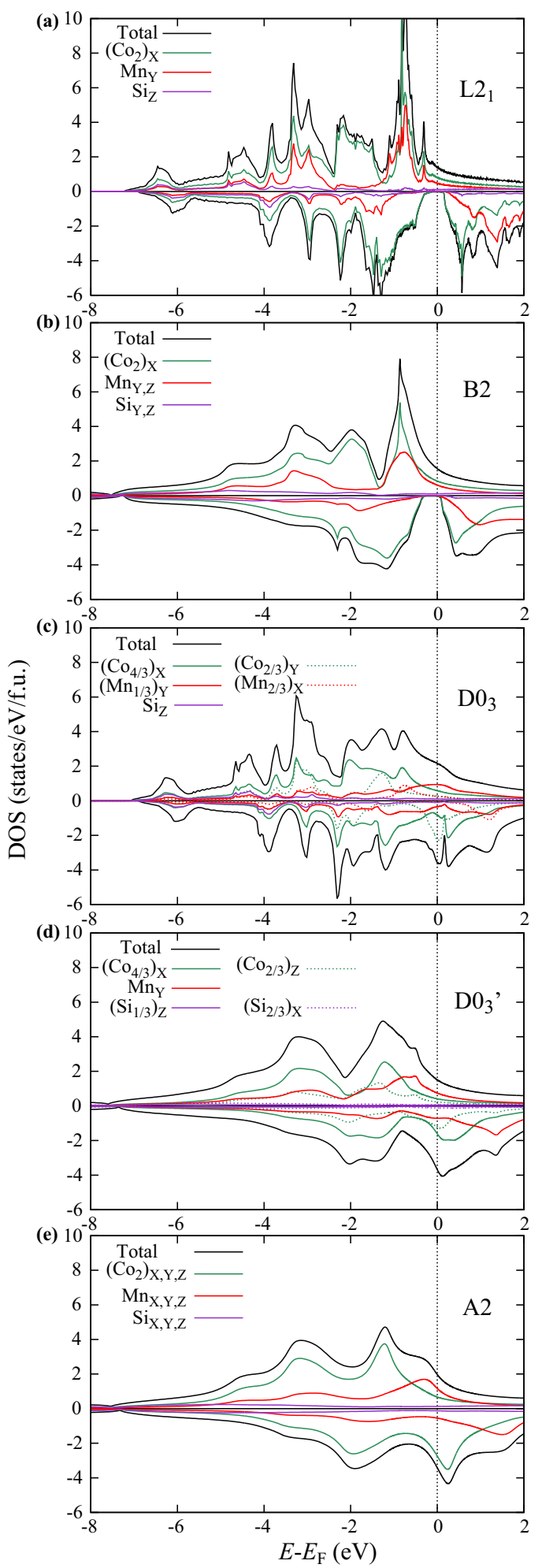

FIG. 1. $\mathrm{Co}_{2} \mathrm{MnSi}$ total DOS and atomic- and site-dependent contributions, calculated with $a_{0}^{\exp }$ for (a) the $\mathrm{L} 2{ }_{1}$, (b) the B2, (c) the $\mathrm{DO}_{3}$, (d) the $\mathrm{DO}_{3}$ ', and (e) the $\mathrm{A} 2$ phases. The upper and lower parts of each panel respectively show the majority and minority spin DOS.

species with the largest magnetic moment $\left(\approx 3 \mu_{B} /\right.$ f.u. $)$, followed by Co $\left(\approx 2 \mu_{B} /\right.$ f.u. $)$.

The Gilbert damping parameter that we calculated for the nearly ideal L2 1 phase takes a value of $1.5 \times 10^{-4}$, which is

of the same order of magnitude than those, reported in the literature, calculated for the perfectly ordered $\mathrm{L} 2$ phase using the BSF or the TCM methods (see Table I). At this point, we conclude that our calculations have correctly recovered the theoretically predicted ideal properties of $\mathrm{Co}_{2} \mathrm{MnSi}$ : the perfectly ordered and cubic L 21 phase possesses a half-metallic character, a ferromagnetic ordering and a low magnetizationprecession damping parameter. In the next sections, we will see how deviations from the perfect structure can worsen these properties.

\section{INFLUENCE OF THE CHEMICAL DISORDER}

\section{A. Disorder dependence of the ground-state energy}

The ground-state energy $E_{0}$ of $\mathrm{Co}_{2} \mathrm{MnSi}$ is plotted in Fig. 2 as a function of the disorder parameters $(x, y, z)$. The ordered L2 1 phase has the lowest ground-state energy $E_{0}(0,0,0)$. In Fig. 2(a), we observe an increase of $E_{0}$ of about $0.55 \mathrm{eV}$ between the $\mathrm{L} 2{ }_{1}$ and the $\mathrm{D}_{3}$ phases, when $a_{0}^{\exp }$ is used. The energy rise is smaller $(\approx 0.352 \mathrm{eV})$ between the $\mathrm{L} 2_{1}$ and the B2 phases, as shown in Fig. 2(b).

Crystal phases involving $\mathrm{Co} / \mathrm{Si}$ swaps (A2-like or $\mathrm{DO}_{3}$ 'like partial disorders) give rise to rapid increases of the ground-state energy as a function of the disorder rate. The fully disordered $\mathrm{A} 2$ and $\mathrm{DO}_{3}$ ' phases have nearly the same energy, approximately $1.45 \mathrm{eV} / \mathrm{f}$.u. higher than $E_{0}(0,0,0)$ and $1 \mathrm{eV} / \mathrm{f}$.u. higher than the energy of the perfectly disordered $\mathrm{B} 2$ or $\mathrm{DO}_{3}$ phases [see Figs. 2(a) and 2(c)]. The comparison between the ground-state energies of the perfectly ordered or disordered phases shows that the $\mathrm{L} 2$ ordered crystal should be the most stable, followed by the $\mathrm{B} 2$ phase and the $\mathrm{DO}_{3}$ phase which is slightly less favorable than the B2 phase. The A2 and $\mathrm{DO}_{3}$ ' structures are the most unlikely fully disordered phases of $\mathrm{Co}_{2} \mathrm{MnSi}$ and should not be observed experimentally. This is in agreement with the previous experimental observations mentioned in Refs. [30,33]. It also explains why the $\mathrm{DO}_{3}$,

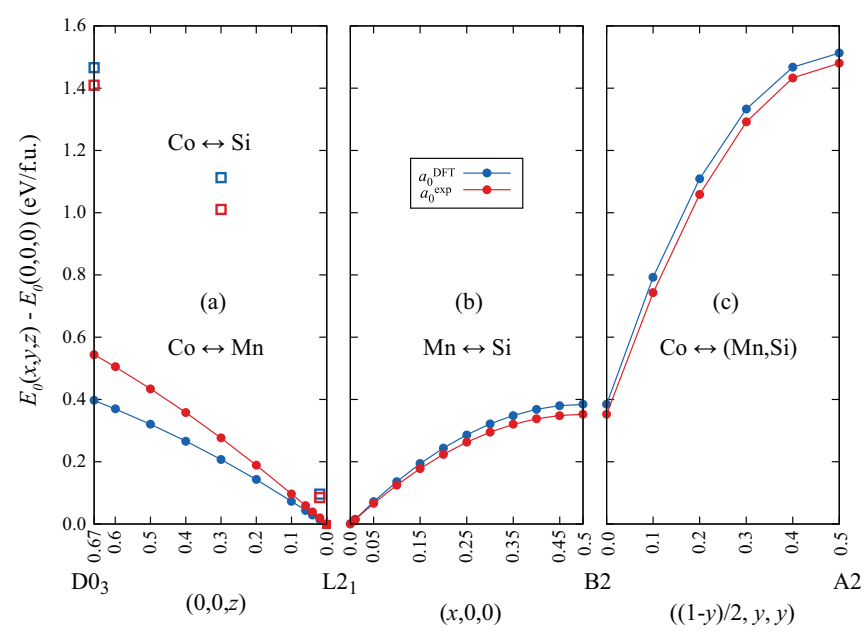

FIG. 2. Ground-state energy $E_{0}$ of $\mathrm{Co}_{2} \mathrm{MnSi}$ per f.u. as a function of the disorder rates $(x, y, z)$, for the atomic distributions described in Sec. II and for the two values of the lattice parameter $a_{0}^{\exp }$ and $a_{0}^{\mathrm{DFT}}$. The ground-state energy for $\mathrm{D}_{3}$ '-like disorder is also plotted (with open squares) in panel a) for $z=0.02,1 / 3$, and $2 / 3$. The energy of the $\mathrm{L} 2$ phase $E_{0}(0,0,0)$ is taken as a reference. 
phase has not been reported in the literature: if $\mathrm{Co} / \mathrm{Si}$ swaps are present in a sample, $\mathrm{Co} / \mathrm{Mn}$ and $\mathrm{Mn} / \mathrm{Si}$ swaps, which are easier to form, will probably also be present in the same sample.

The stability rank of the disordered phases may however change if we consider partial, instead of perfect disorders. This is in particular the case for the partial $\mathrm{D}_{3}$-like and $\mathrm{B} 2$-like disorders: a comparison between the ground-state energies of these two phases for disorder rates involving the same number of Mn atom swaps $(x=z)$ shows that $E_{0}(0,0, z)<E_{0}(x, 0,0)$ for $z=x<0.35$ when the experimental lattice parameter is used. This could be the reason why the partial $\mathrm{D}_{3}$-like disorder has been observed in real samples.

\section{B. Disorder dependence of the electronic structure}

The electron states of the $\mathrm{Co}_{2} \mathrm{MnSi}$ crystal are strongly modified by the different kinds of atom disorder that may exist in this alloy. This can be seen in Fig. 1(b) to Fig. 1(e), where we have plotted the total DOS, together with the contributions of the different chemical species and atomic sites, for all the perfectly disordered crystal phases $\left(\mathrm{B} 2, \mathrm{D}_{3}, \mathrm{DO}_{3}{ }^{\prime}\right.$, and $\left.\mathrm{A} 2\right)$, as calculated using $a_{0}^{\exp }$.

A comparison between these results and those shown in Fig. 1(a) for the perfectly ordered $\mathrm{L} 2{ }_{1}$ phase shows that disorder tends to globally smooth the DOS curves, and destroys most of the sharp peaks calculated for the $\mathrm{L} 2_{1}$ phase. Disorder only slightly narrows the minority spin band gap for the perfectly disordered $\mathrm{B} 2$ phase. This does not affect the half-metallic character of $\mathrm{Co}_{2} \mathrm{MnSi}$ when $a_{0}^{\exp }$ is used to calculate the electronic structure. For all the other perfectly disordered phases (see Fig. 1), the half-metallic character is unambiguously destroyed. This is due to the fact that these disordered structures all involve swaps of Co with other chemical species atoms, modifying the first coordination shell of all the atomic sites and resulting in the appearance of new electron states in the energy range where the minority spin band gap was located for the $\mathrm{L} 2{ }_{1}$ phase. The new minority spin electron states which appear at the Fermi level are mainly built from the $d$ orbitals of Co atoms in $\mathrm{Y}$ atomic sites for the $\mathrm{D}_{3}$ phase [see Fig. 1(c)], in agreement with former calculations performed by Picozzi et al. [34], and are built from $d$ orbitals of Co atoms in both $\mathrm{X}$ and $\mathrm{Z}$ sites (and also of Mn atoms) for the $\mathrm{D}_{3}$ ' phase [Fig. 1(d)] and of all the Co and Mn atoms for the A2 phase [Fig. 1(e)].

We will now discuss the modification of the electron states induced by the different kinds of partial disorder in $\mathrm{Co}_{2} \mathrm{MnSi}$. The most important consequence of atom disorder being the appearance of new electron states that may destroy the halfmetallicity of $\mathrm{Co}_{2} \mathrm{MnSi}$, we will focus on the variation of the density of majority and minority spin states at the Fermi level, $n^{\uparrow}\left(E_{F}\right)$ and $n^{\downarrow}\left(E_{F}\right)$, which are displayed in Fig. 3 as a function of $(x, y, z)$. For a B2-like partial disorder, the half-metallic character of $\mathrm{Co}_{2} \mathrm{MnSi}$ is, of course, preserved for all disorder rates $x$ when the experimental lattice parameter is used.

Partial disorders involving $\mathrm{Mn} / \mathrm{Co}$ atomic swaps have stronger consequences on the density of states at the Fermi level. The electron states induced by a $\mathrm{D}_{3}$-like disorder at energies within the minority spin band gap of the $\mathrm{L} 2_{1}$ phase appear even for tiny disorder rates. Whatever the value of $z$, some of these states can be found at the Fermi level and

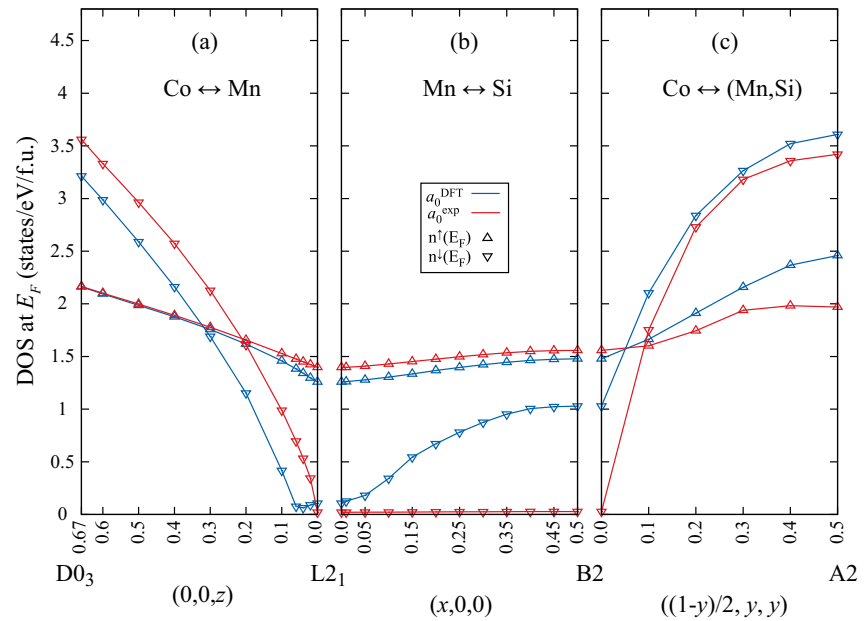

FIG. 3. Majority- and minority-spin DOS at the Fermi level $\left[n^{\uparrow}\left(E_{F}\right)\right.$ and $n^{\downarrow}\left(E_{F}\right)$, respectively] as a function of the disorder rates $(x, y, z)$ for the two values of the lattice parameter.

destroy the half-metallic character of $\mathrm{Co}_{2} \mathrm{MnSi}$ when $a_{0}^{\exp }$ is used, as shown in see Fig. 3(a). They are responsible for a strong increase of $n^{\downarrow}\left(E_{F}\right)$ when the disorder rate $z$ increases. It follows that the spin polarization at the Fermi level $P\left(E_{F}\right)=\frac{n^{\uparrow}\left(E_{F}\right)-n^{\downarrow}\left(E_{F}\right)}{n^{\uparrow}\left(E_{F}\right)+n^{\downarrow}\left(E_{F}\right)}$ changes its sign for $z \approx 0.2$. A similar strong increase of $n^{\downarrow}\left(E_{F}\right)$ can be observed for an A2-like partial disorder, for which the spin-polarization at the Fermi level is inverted for $y>0.1$ when $a_{0}^{\exp }$ is used, see Fig. 3(c). The half-metallic character of $\mathrm{Co}_{2} \mathrm{MnSi}$ is thus strongly weakened by $\mathrm{A} 2$-like or $\mathrm{D}_{3}$-like partial disorders. The definition of the spin polarization at the Fermi level given in this section should be suitable for describing data deduced from photoemission spectra (at least for describing the contribution of bulklike states to the spin polarization [27]); surface states and surface resonances should also contribute to the photoemission spectra. Another definition of the spin polarization [46] should probably be more suitable to analyze the spin polarization at the Fermi level deduced from magnetotransport measurements.

\section{Disorder dependence of the static magnetic properties}

The total (spin+orbital) magnetic moment per $\mathrm{Co}_{2} \mathrm{MnSi}$ f.u. is shown in Fig. 4 as a function of the disorder rates $(x, y, z)$. While it does not vary significantly for B2-like partial disorders [Fig. 4(b)], the total magnetic moment calculated with $a_{0}^{\exp }$ decreases linearly with $z$ for $\mathrm{D}_{3}$-like disorders, from $\simeq 5 \mu_{B}$ for the perfectly ordered $\mathrm{L} 2_{1}$ to $\simeq 3.35 \mu_{B}$ for the perfectly disordered $\mathrm{D}_{3}$ phases [Fig. 4(a)]. This decrease is in agreement with the results reported by Picozzi et al. [34], who found a total spin magnetic moment of $4.5 \mu_{B}$ /f.u., using a supercell with $z=0.125$. Figure 5 shows that this strong decrease of the magnetization is mainly due to a reduction of the averaged spin magnetic moment of $\mathrm{Mn}$ atoms, which is $2 \mu_{B}$ lower in the $\mathrm{D}_{3}$ than in the $\mathrm{L} 2_{1}$ phase.

The results shown in Fig. 5 correspond to an average of the spin magnetic moments calculated, for each chemical species on the different atomic sites of the crystal. They can take disparate values depending on the atomic sites (not shown in 


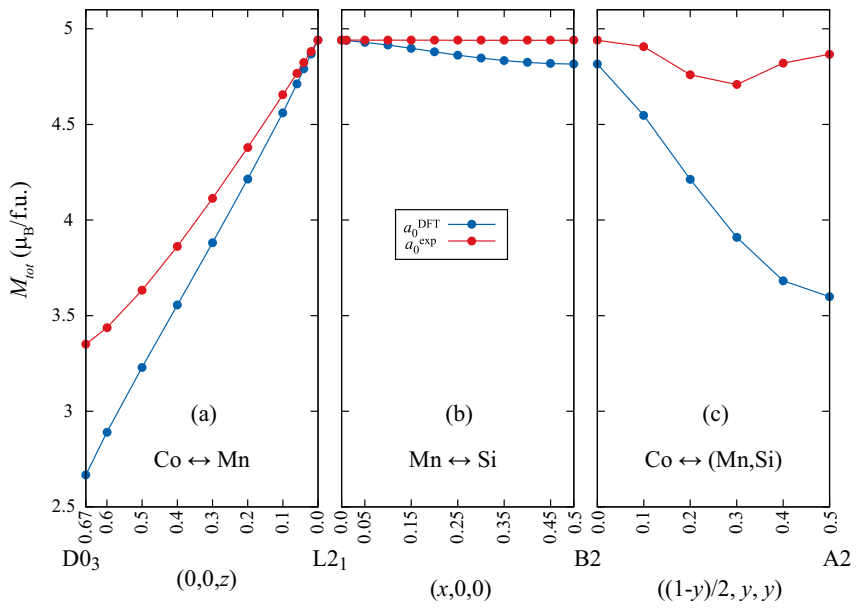

FIG. 4. Total magnetic moment $M_{\text {tot }}$ per formula unit as a function of the disorder rates $(x, y, z)$ and calculated with the two values of the lattice parameter.

the figures), in particular for $\mathrm{Mn}$ atoms. The spin magnetic moment of $\mathrm{Mn}$ atoms located in the $\mathrm{Y}$ sites is high and takes values rather close to that calculated for the $\mathrm{L} 22_{1}$ phase, only decreasing from 3.02 to $2.58 \mu_{B}$ when $z$ increases from 0 to $\frac{2}{3}$, when $a_{0}^{\exp }$ is used. Conversely, the spin magnetic moment of $\mathrm{Mn}$ atoms located in $\mathrm{X}$ atomic sites is smaller, varying from $-0.75 \mu_{B}$ for $z=0.02$ to $0.19 \mu_{B}$ for $z=\frac{2}{3}$. The strong decrease of the averaged Mn spin magnetic moment is thus mainly due to the increasing number of Mn atoms with a small magnetic moment when the $\mathrm{DO}_{3}$-like disorder increases.

We found an antiferromagnetic ordering between the magnetic moments of $\mathrm{Mn}$ atoms in $\mathrm{X}$ and $\mathrm{Y}$ atomic sites for low $\mathrm{D}_{3}$-like disorder rates; it however disappears for higher disorder rates $\left(z \geqslant 0.54\right.$ when $a_{0}^{\exp }$ is used). The origin of this antiferromagnetic ordering can be understood as follows: in the perfectly ordered L2 1 phase, Co and Mn atoms are respectively located in the $\mathrm{X}$ and $\mathrm{Y}$ atomic sites, and the smallest distance between these atoms is given by

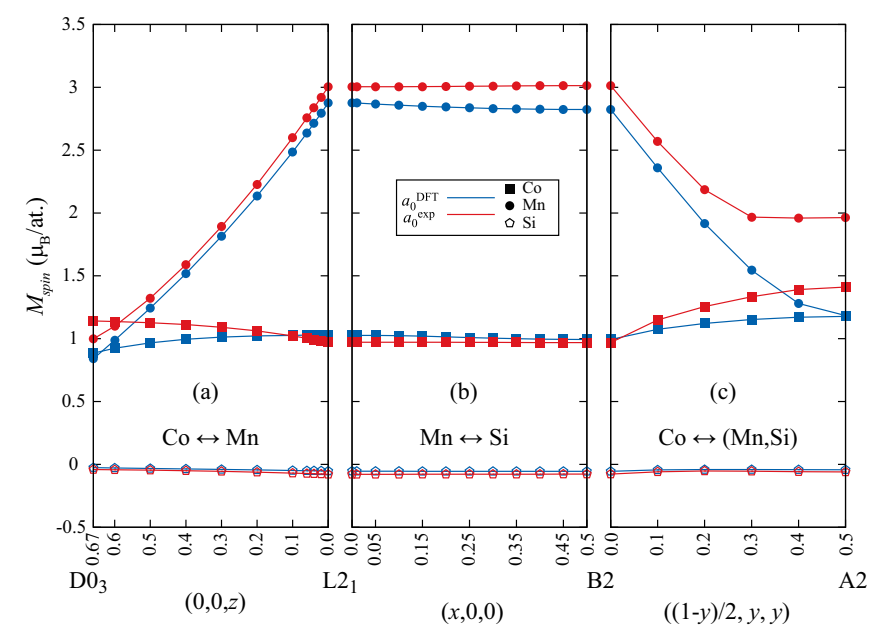

FIG. 5. Averaged value of the spin magnetic moment $M_{\text {spin }}$ of the different atoms, as a function of the disorder rates $(x, y, z)$ and calculated with the two values of the lattice parameter.
$d_{\mathrm{CoMn}} \approx 0.433 a_{0}, d_{\mathrm{CoCo}}=0.5 a_{0}$, and $d_{\mathrm{MnMn}} \approx 0.707 a_{0}$, leading to a strong ferromagnetic coupling between spin magnetic moments of $\mathrm{Mn}$ and $\mathrm{Co}$ atoms, and to smaller ferromagnetic coupling between $\mathrm{Co}$ atoms and between $\mathrm{Mn}$ atoms [59]. A swap between $\mathrm{Mn}$ and $\mathrm{Co}$ atoms reduces some of these distances, which can become as small as $d_{\mathrm{MnMn}} \approx 0.433 a_{0}$ or $d_{\text {CoCo }} \approx 0.433 a_{0}$, respectively, between two Mn and two Co atoms located on first neighbor $\mathrm{X}$ and $\mathrm{Y}$ atomic sites. It follows, according to the Bethe-Slater curve, which describes the magnetic coupling as a function of the distance between magnetic atoms, that the magnetic moments of Mn atoms located on nearest-neighbor $\mathrm{X}$ and $\mathrm{Y}$ atomic sites become antiferromagnetically coupled. The magnetic structure of the alloy and its actual magnetic ordering finally results from the competition between the same ferromagnetic coupling as those previously listed for the $\mathrm{L} 2{ }_{1}$ phase, and new antiferromagnetic coupling between atoms, due to the $\mathrm{DO}_{3}$-like disorder. These competing interactions could be the reason why $\mathrm{Mn}$ atoms have a much smaller spin magnetic moment in $\mathrm{X}$ than in $\mathrm{Y}$ atomic sites.

The magnetic ordering depends on the alloy disorder rate, since the averaged numbers of first neighbor $\mathrm{Co}_{\mathrm{X}} / \mathrm{Co}_{Y}$, $\mathrm{Co}_{\mathrm{X}} / \mathrm{Mn}_{\mathrm{Y}}, \mathrm{Mn}_{\mathrm{X}} / \mathrm{Mn}_{\mathrm{Y}}$ atoms strongly depend on $z$; in particular, the number of $\mathrm{Mn}_{Y}$ atoms first neighbor of a given swapped $\mathrm{Mn}_{\mathrm{X}}$ atom should decrease when $z$ increases. This could explain why the ordering between the magnetic moments of $\mathrm{Mn}$ atoms located in $\mathrm{X}$ and $\mathrm{Y}$ sites switches from antiferromagnetic to ferromagnetic when the disorder rate $z$ increases.

The results described above are quantitatively in agreement with those previously described by Picozzi et al. [34] who considered a single $\mathrm{Co} / \mathrm{Mn}$ swap inside a rather big supercell (such a configuration corresponds to a low value of $z$ ). They calculated an antiferromagnetic coupling between the spin magnetic moments of the $\mathrm{Mn}$ atom located in a $\mathrm{X}$ atomic site $\left(-0.83 \mu_{B}\right.$ while we obtain $\left.-0.75 \mu_{B}\right)$ and of the other magnetic atoms. The fact that the magnetic order of a complex alloy depends on the atom disorder has been studied previously for several full Heusler alloys like $\mathrm{Co}_{2} \mathrm{CrAl}$ [60], $\mathrm{Ni}_{2} \mathrm{MnAl}$ [61], $\mathrm{Ni}_{2} \mathrm{MnGa}$ [62], $\mathrm{Fe}_{2} \mathrm{VAl}$ [63], and $\mathrm{MnCo}$ [64-66].

For partial disorders intermediate between the B2 and the A2 phases [Fig. 4(c)], the total magnetic moment does not show a monotonous behavior when the experimental lattice constant is considered. This can be analyzed in more details by considering the disorder dependence of the averaged spin magnetic moment of the different atoms, as we can see in Fig. 5: the decrease of the spin magnetic moment of Mn atom is higher than the increase of the spin magnetic moment of Co atoms when $y$ varies from 0.0 to 0.3 , which explains that the total magnetic moment decreases for these disorder rates. Conversely, the spin magnetic moment is constant for $\mathrm{Mn}$ atoms, while it increases for Co atoms when $y \geqslant 0.3$, which explains that the total magnetic moment increases when the disorder rate approaches that of the $\mathrm{A} 2$ phase. As for $\mathrm{DO}_{3}$ like partial disorders, the disorder rate dependence of the spin magnetic moments of $\mathrm{Co}, \mathrm{Mn}$, and $\mathrm{Si}$ atoms shown in Fig. 5 corresponds to an average of the magnetic moments calculated for the different atomic sites. Again, the spin magnetic moment of $\mathrm{Mn}$ atoms located on $\mathrm{X}$ atomic sites is small and negative 


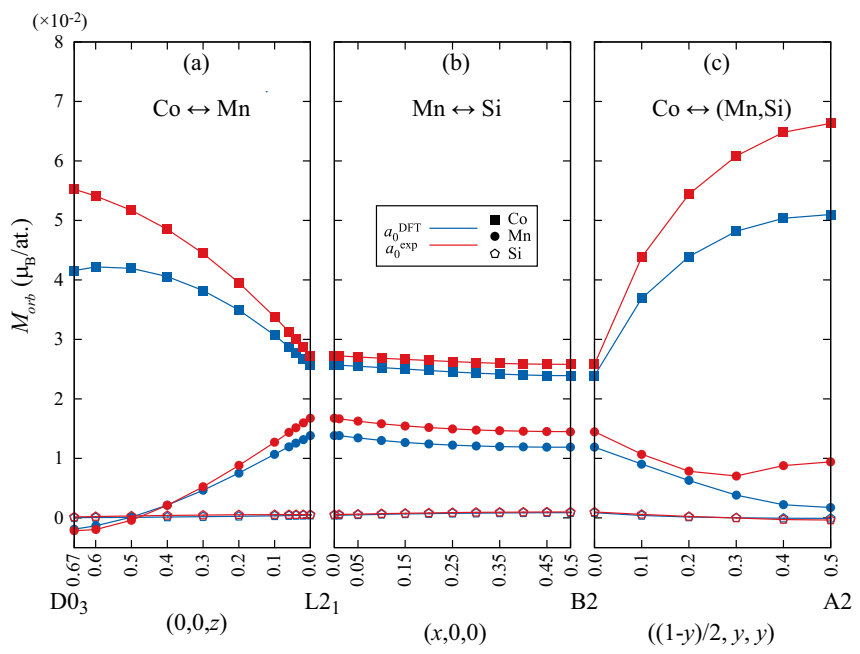

FIG. 6. Averaged value of the orbital magnetic moment $M_{\text {orb }}$ of the different atoms, as a function of the disorder rates $(x, y, z)$ and calculated with the two values of the lattice parameter.

for very low disorder rates. This antiferromagnetic ordering rapidly disappears when the disorder increases. A microscopic interpretation in terms of ferromagnetic and antiferromagnetic coupling is more complicated here, as the complex variations of the magnetic moments are now governed by both $\mathrm{Co} / \mathrm{Mn}$ and $\mathrm{Co} / \mathrm{Si}$ swaps.

$\mathrm{X}$-ray magnetic circular dichroism experiments have already been performed on $\mathrm{Co}_{2} \mathrm{MnSi}$ samples $[58,67,68]$ and have been used to extract the values $M_{\text {spin }}=0.96 \mu_{B}$ and $M_{\text {orb }}=0.034 \mu_{B}$ for Co atoms, and $M_{\text {spin }}=2.59 \mu_{B}$ and $M_{\text {orb }}=0.020 \mu_{B}$ for Mn atoms [58]. The differences between these measured magnetic moments and those we computed for the L2 $2_{1}$ phase using $a_{0}^{\exp }$, can of course be explained by the discrepancy usually observed between numerical results based on the DFT and experimental measurements; they can also be interpreted, according to the curves shown in Figs. 5 and 6 , as a possible indication of a small $\mathrm{D}_{3}$-like (with $z \approx 0.1$ ) or A2-like disorder in the samples considered in Ref. [58]. Note, however, that the comparison between magnetic moments calculated with DFT-based methods and measured by XMCD techniques is not trivial and must be considered with caution, due to the limitations of XMCD sum rules for measuring magnetic moments [69-71]. In particular, important parameters that enter XMCD sum rules are not known and can only be estimated (this is the case of the number of holes in the $d$ bands) or neglected (the magnetic dipole operator $\mathrm{T}_{z}$ ). Moreover, the fact that the measured orbital magnetic moment of $\mathrm{Mn}$ atoms is slightly higher than our computed values is not surprising because DFT-based methods are known to better estimate the spin than the orbital magnetic moments of transition metal atoms. Nevertheless, our calculations on spin and orbital magnetic moments provide additional sets of data on the static magnetic properties, that could be used independently one from the others to investigate the consequences of complex partial disorders in such alloys.

The Landé factor $g$ is also an interesting parameter that gives information on the ratio of orbital to spin magnetic moments. In weak ferromagnets, we have $(g-2)=2 M_{\text {orb }} / M_{\text {spin }}$ where $M_{\text {orb }}$ and $M_{\text {spin }}$ are the orbital and spin magnetic moments per formula unit. In addition, $g$ is particularly important for spin dynamic measurements as it fits the ferromagnetic resonance and spin wave frequencies of magnetic materials. Indeed, it is used to define the gyromagnetic ratio $\gamma$ entering the Landau-Lifshitz equation as $\gamma=\frac{g \times|e|}{2 m_{e}}$, where $e$ and $m_{e}$ are the electron charge and mass. The results presented in Figs. 5 and 6 can be used to calculate the dependence of the Landé factor with alloy disorder. We found that $(g-2)$ takes the values $0.029,0.067,0.027$, and 0.060 , respectively, for the $\mathrm{L} 22_{1}$, $\mathrm{DO}_{3}, \mathrm{~B} 2$, and $\mathrm{A} 2$ phases of $\mathrm{Co}_{2} \mathrm{MnSi}$, when the experimental lattice parameter is used. Values of the Landé factor measured in experiments for $\mathrm{Co}_{2} \mathrm{MnSi}$ samples are ranging from 2.00 to 2.06, according to Refs. [72-74]. These values are of the same order of magnitude as our calculated data. Other values of the $g$ factor have been extracted from FMR measurements performed on several samples with different disorder rates and are presented in details in Ref. [75]. The Landé $g$ factor increases with $\mathrm{Co} / \mathrm{Mn}$ swaps in agreement with the increase of the orbital moment induced by this kind of disorder. $\mathrm{L} 2_{1}$ and B2 crystal orders show similar values of the Landé factor, in agreement with the orbital and spin magnetic moments calculated for these two phases.

\section{Disorder dependence of the Gilbert damping parameter $\alpha$}

Our results for the Gilbert damping parameter of the perfectly disordered $\mathrm{B} 2, \mathrm{D}_{3}$, and $\mathrm{A} 2$ phases are reported in Table II, where they are compared to theoretical results found in the literature (we did not find any calculated value of $\alpha$ for the $\mathrm{D}_{3}$ phase). The agreement is very good between the Gilbert damping parameters of the A2 and B2 phases that we obtained using the linear response formalism and the electronic structure calculated from first principles, and the results obtained from the TCM and a tight-binding description of the electron states.

The values of the Gilbert damping parameter $\alpha$ calculated for partial disorders intermediate between those of the $\mathrm{L} 2_{1}$, $\mathrm{D}_{3}, \mathrm{~B} 2$, and $\mathrm{A} 2$ phases of $\mathrm{Co}_{2} \mathrm{MnSi}$ are shown in Fig. 7, where they are represented as a function of the disorder rates $(x, y, z)$ and for the two values of the lattice parameter. According to Fig. 7(b), $\alpha$ is nearly independent of the B2-like

TABLE II. Gilbert damping parameter $\alpha$ of the $\mathrm{B} 2, \mathrm{~A} 2$, and $\mathrm{D}_{3}$ phases of $\mathrm{Co}_{2} \mathrm{MnSi}$, calculated in the present paper and compared to theoretical results from the literature.

\begin{tabular}{|c|c|c|c|c|}
\hline & $a_{0}(\AA)$ & $\alpha_{\mathrm{B} 2}$ & $\alpha_{\mathrm{A} 2}$ & $\alpha_{\mathrm{D} 0_{3}}$ \\
\hline Present paper, KKR, LSDA, LRF & 5.48 (calc) & $6.3 \times 10^{-4}$ & $5.2 \times 10^{-3}$ & $7.4 \times 10^{-3}$ \\
\hline Present paper, KKR, LSDA, LRF & $5.65(\exp )$ & $1.9 \times 10^{-4}$ & $3.2 \times 10^{-3}$ & $5.2 \times 10^{-3}$ \\
\hline KKR, tight-binding, TCM [25] & $5.65(\exp )$ & $2.0 \times 10^{-4}$ & $3.5 \times 10^{-3}$ & - \\
\hline
\end{tabular}




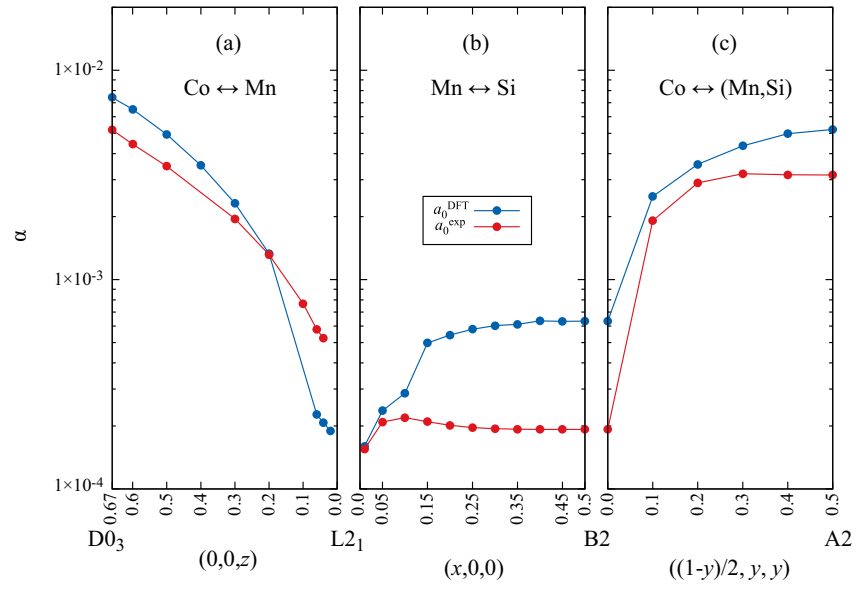

FIG. 7. Gilbert damping parameter $\alpha$ as a function of the disorder rates $(x, y, z)$ and calculated with the two values of the lattice parameter.

disorder rate when $a_{0}^{\exp }$ is used. Figures 7(a) and 7(c) show that Co-Mn swaps have a more important effect, inducing a strong increase of $\alpha$. When $a_{0}^{\exp }$ is used, the calculated Gilbert damping parameter is respectively 32 and 19 times higher for the $\mathrm{D}_{3}$ and $\mathrm{A} 2$ phase, than for the nearly $\mathrm{L} 2_{1}$ crystal. This strong increase of $\alpha$ yet occurs for small $\mathrm{D}_{3}$-like or A2-like disorder rates, and a small amount of Co-Mn swaps is sufficient to strongly increase the damping.

\section{E. Influence of the lattice parameter on the disorder dependence of the physical properties}

The position of the Fermi energy within the minority spin band gap strongly depends on the value of the lattice parameter. While the Fermi level of the $\mathrm{L} 2_{1}$ phase was located near the center of this band gap for $a_{0}^{\exp }$, it shifts closer to the minimum of the minority-spin conduction bands when $a_{0}^{\mathrm{DFT}}$ is used (see Fig. 8). This does not affect the half-metallic character of $\mathrm{Co}_{2} \mathrm{MnSi}$ for the ordered $\mathrm{L} 22_{1}$ phase, but has stronger consequences for B2-like disordered alloys

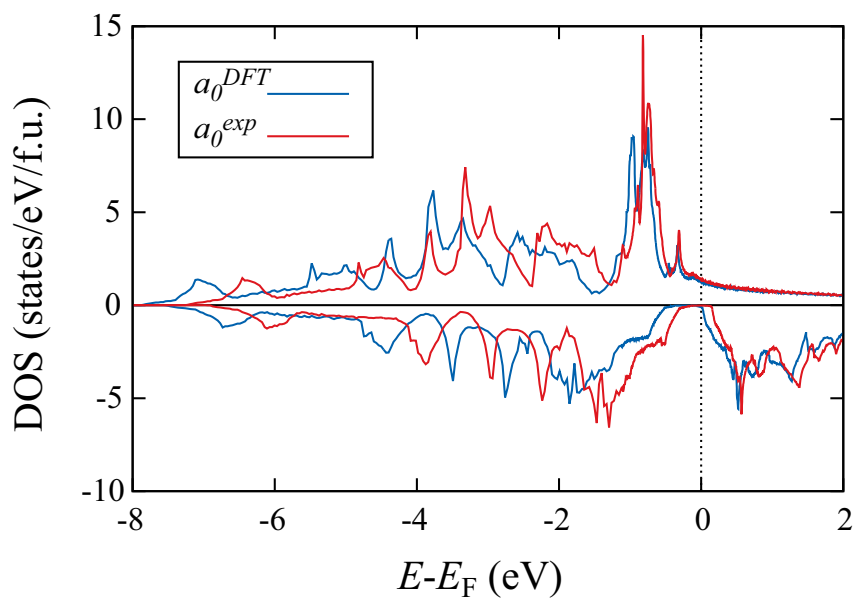

FIG. 8. Total DOS of $\mathrm{Co}_{2} \mathrm{MnSi}$, calculated for the $\mathrm{L} 2{ }_{1}$ phase with $a_{0}^{\exp }$ (red curve) and $a_{0}^{\mathrm{DFT}}$ (blue curve). when $a_{0}^{\mathrm{DFT}}$ is used: in this case, the disorder broadens the edge of the minority spin unoccupied band edge, which was just above the Fermi energy for the perfectly ordered $\mathrm{L} 2_{1}$ phase. The tail of the corresponding minority spin continuum thus slightly crosses the Fermi level, destroying the halfmetallicity $\left(n^{\downarrow}\left(E_{F}\right) \neq 0\right)$, even at small disorder rates [see Fig. 3(b)]. This has for consequences to increase the total DOS $Z_{F}=\left[n^{\uparrow}\left(E_{F}\right)+n^{\downarrow}\left(E_{F}\right)\right]$ and to reduce in the same time the spin-polarization at the Fermi level $P\left(E_{F}\right)$, when $a_{0}^{\mathrm{DFT}}$ is used.

Using $a_{0}^{\mathrm{DFT}}$ instead of $a_{0}^{\exp }$ has also important consequences for $\mathrm{D}_{3}$-like partial disorders: electron states induced in the minority spin band gap by this kind of disorder have energies below $E_{F}$ for very low disorder rates and only cross the Fermi level for $z>0.06$ when $a_{0}^{\mathrm{DFT}}$ is used, while the same kind of states were found at the Fermi level even for very small D03-like disorder rates for $a_{0}^{\text {exp }}$, see Fig. 3(a). Using $a_{0}^{\mathrm{DFT}}$, the spin-polarization at the Fermi level is of $100 \%$ for $0 \leqslant z \leqslant 0.06$, strongly decreases for $0.06 \leqslant z \leqslant 0.3$ and finally changes its sign for $z \approx 0.3 . a_{0}^{\mathrm{DFT}}$ being smaller than $a_{0}^{\exp }$, a simple comparison between the ground-state energies calculated for these two lattice parameters and the same D03-like disorder rate (see Fig. 2) also shows that a small isotropic compression of the crystal, which may for instance occur for $\mathrm{Co}_{2} \mathrm{MnSi}$ particles embedded in a matrix with a slightly smaller lattice parameter, favors $\mathrm{Mn} / \mathrm{Co}$ swaps (without significantly favoring other kinds of atomic swaps).

The total magnetic moment strongly depends on the lattice parameter for partial disorders intermediate between the B2 and the A2 phases [Fig. 4(c)]; while it did not show a monotonous behavior for $a_{0}^{\exp }$, it decreases almost linearly with $y$ when $a_{0}^{\mathrm{DFT}}$ is used. The difference between these two behaviours can be explained as follows: between the $\mathrm{B} 2$ and the A2 phases, the disorder-induced decrease of the spin magnetic moment of Mn atoms is smaller by $0.78 \mu_{B}$ with $a_{0}^{\exp }$ than with $a_{0}^{\mathrm{DFT}}$, while the increase of the magnetic moment of Co atoms is higher by $0.24 \mu_{B}$. The sum of the two main contributions (Co and Mn spin magnetic moments) thus provides an almost monotonous decay when $a_{0}^{\mathrm{DFT}}$ is used.

According to Fig. 7(b), $\alpha$ increases more strongly (from $1.6 \times 10^{-4}$ to $6.3 \times 10^{-4}$ ) with the B2-like disorder rate when $a_{0}^{\mathrm{DFT}}$ is used, instead of $a_{0}^{\exp }$. Figure 7 also shows that the Gilbert damping parameter is in general higher for $a_{0}^{\mathrm{DFT}}$ then for $a_{0}^{\exp }$ (except for small D0 $0_{3}$-like disorder rates), which means that a small isotropic compression of the crystal generally tends to increase the magnetic damping.

\section{DISCUSSION}

In the previous sections, we have seen the influence of partial atomic disorders on the main electronic and magnetic properties of $\mathrm{Co}_{2} \mathrm{MnSi}$. We can make some correlations between the different quantities which characterize the physical properties of $\mathrm{Co}_{2} \mathrm{MnSi}$, using the expressions of the damping parameter proposed by Kamberský [36] in his attempt to describe the dissipation of the magnetization in magnetic metallic materials. According to Kamberský, the magnetic damping parameter can be described by the two following 
contributions:

$$
\alpha_{\mathrm{sf}}=\frac{\pi \gamma \hbar^{2}}{\mu_{0} M} Z_{F} \frac{(g-2)^{2}}{\tau},
$$

which holds for spin-flip scattering contributions; it roughly accounts for the interband terms of the torque-correlation model, and

$$
\alpha_{\mathrm{o}}=\frac{\pi \gamma}{\mu_{0} M} Z_{F} \lambda_{\mathrm{SO}}^{2}(g-2)^{2} \tau,
$$

which describes ordinary scattering. This latter contribution roughly accounts for the intraband terms of the torque correlation model. Both terms result from the interaction between the defects of the lattice and the spin of the itinerant electrons through spin-orbit coupling. In these equations, $\gamma$ is the gyromagnetic ratio, $M$ the saturation magnetization (here, directly proportional to the total magnetic moment since we have shown that the volume of the unit cell does not significantly change with disorder), $Z_{F}$ the DOS at the Fermi level, $\lambda_{\text {SO }}$ the spin-orbit coupling parameter, $g$ the Landé factor and $\tau$ the electron scattering time. The variations of $\alpha$ with chemical disorder can then be mostly explained and related to the electronic structure of the alloy. Indeed, as pointed out by Mankovsky et al. [51] or by Kamberský [36], $Z_{F}$ can be considered as a measure of the number of available magnetic relaxation channels. The small damping parameter calculated for the $\mathrm{L} 2{ }_{1}$ phase of $\mathrm{Co}_{2} \mathrm{MnSi}$ originates from the half-metallic character of this alloy, as previously mentioned by Liu et al. [24]. Half-metallicity shall in particular prevent from spin-flip relaxation processes.

The Mn/Co swaps which characterize $\mathrm{DO}_{3}$-like disorders create new Co $d$ states at the Fermi level, which correspond to intense peaks in the minority spin DOS curve [see Fig. 1(c)] and to the creation of new relaxation channels that allow the magnetization to dissipate faster. It follows that the Gilbert damping parameter increases strongly for a $\mathrm{DO}_{3}$-like disordered alloy. The increase of $\alpha$ as a function of the disorder rate $z$ is similar to the increase of the density of states at the Fermi level, as we can see by comparing Figs. 3(a) and 7(a).

This kind of analysis can be extended to B2 partial disorders. If we first focus on the results obtained with $a_{0}^{\mathrm{DFT}}$, we observe a similar increase of $\alpha$ and $Z_{F}$ with the disorder rate $x$ [compare Figs. 3(b) and 7(b)]. In particular, the disappearance of the half-metallicity resulting from the small overlap of the minority spin conduction band edge corresponds to an increase of $\alpha$. This increase is, however, lower than for $\mathrm{DO}_{3}$ partial disorders. This can be explained by the fact that the disorder-induced minority spin DOS at the Fermi level does not involve Co atoms, but only the $\mathrm{Mn}$ and $\mathrm{Si}$ atoms of their first coordination shell, resulting in a diffuse minority spin conduction band edge due to $\mathrm{Mn} / \mathrm{Si}$ atomic swaps. This diffuse conduction band edge is at energies high enough above $E_{F}$ to preserve the half-metallic character of the alloy, when $a_{0}^{\exp }$ is used; this explains why the Gilbert damping parameter is smaller for this lattice parameter for which it only slightly increases from $1.5 \times 10^{-4}$ to $1.9 \times 10^{-4}$, mostly because the potential scattering induced by chemical disorder increases with $x$ [76].

The same conclusions can finally be drawn for A2-like disorders, for which the damping parameter $\alpha$ and the DOS at the Fermi level show similar variations as a function of the disorder rate $y$, as we can see by comparing Figs. 3(c) and 7(c). Equations (2) and (3) show that the Gilbert damping parameter not only depends on the DOS at the Fermi level $Z_{F}$, but also on other physical parameters $\left(M, \lambda_{\mathrm{SO}}, g, \gamma\right.$, and $\tau$ ) which characterize the physical properties of $\mathrm{Co}_{2} \mathrm{MnSi}$. As these parameters also depend on the kind of disorder and on the disorder rate, they should also contribute to the $(x, y, z)$ dependence of $\alpha$. A disorder-induced decrease of the total magnetic moment $M_{\text {tot }}$ should, in particular, contribute to an increase of $\alpha$. Figure 3 shows, for instance, that the total DOS $Z_{F}$ is slightly higher for the perfectly disordered A2 phase than for the $\mathrm{DO}_{3}$ phase when $a_{0}^{\mathrm{DFT}}$ is used, while $\alpha$ is found smaller for the $\mathrm{A} 2$ than for the $\mathrm{DO}_{3}$ phase; this can be explained by the fact that $M_{\text {tot }}$ is smaller for the $\mathrm{DO}_{3}$ than for the $\mathrm{A} 2$ disordered crystal (see Figs. 4 and 7). A systematic correlations between $\alpha$ and all the other parameters that characterize $\mathrm{Co}_{2} \mathrm{MnSi}$ is not always easy to establish, in particular because we did not calculate explicitly the disorder rate dependence of the spin-orbit coupling parameter $\lambda_{\text {so }}$ and of the electron scattering time $\tau$.

As specified in Tables I and II, our calculated values for $\alpha$ are in agreement with previous theoretical results computed for the $\mathrm{L} 2{ }_{1}, \mathrm{~A} 2$, or B2 phases. However, most of the experimental values presented in the literature as belonging to the $\mathrm{L} 2{ }_{1}$ phase give values around $4 \times 10^{-3}$ [15-19], more than 10 times higher than our numerical results. Recent experiments gave values of $\alpha$ smaller than $1 \times 10^{-3}$ [20,22,23]. A measured value as small as $1.5 \times 10^{-4}$ has even been reported very recently, which was obtained after a fitting procedure removing the two-magnons scattering contributions to the measured damping [21]. Ferromagnetic resonance measurements were performed in our group on $\mathrm{Co}_{2} \mathrm{MnSi}$ irradiated with light $\mathrm{He}^{+}$ ions, as briefly described in Appendix B. These measurements [75] gave a damping coefficient of $1.7 \times 10^{-3}$ for the ordered L 2 phase and of $6.7 \times 10^{-3}$ when $\mathrm{Co} / \mathrm{Mn}$ swaps are randomly induced in the $\mathrm{L} 21$ phase $(z=0.09)$. This confirms the predictions of an increase of $\alpha$ induced by a $\mathrm{DO}_{3}$-like disorder: in both experimental and theoretical cases, we can observe that $\alpha$ increases by almost a factor 4 between the L 2 phase and the $\mathrm{DO}_{3}$-like phase with a disorder rate of $z \approx 0.09$. The measured values of $\alpha$ however remain well higher than our calculated damping parameter. The ability of experimentalists to continue reporting lower values of $\alpha$ by optimizing the crystal quality is a confirmation that samples with higher values of $\alpha$ certainly contain locally disordered crystal phases with a partial $\mathrm{DO}_{3}$ or (even if this is less probable) a partial A2 disorder, potentially combined with local small variations of the lattice parameter.

In addition to alloy disorder, the difference between experimental and theoretical values of $\alpha$ can also be explained by the fact that the damping measurements are performed at room temperature while our calculations are performed at $0 \mathrm{~K}$, without considering the increase of scattering events resulting from atomic displacement caused by the temperature. A theoretical investigation of these effects has recently been done by Ebert et al. [77]. Temperature-related effects on the Gilbert damping parameter of $\mathrm{Ir}$-doped $\mathrm{Co}_{2} \mathrm{MnSi}$ were studied in Ref. [41] using an alloy-analogy model that accounts for finitetemperature effects via quasistatic random displacements of the atomic sites. As shown in this article, $\alpha$ is influenced by 
the temperature only for the undoped and ordered $\mathrm{Co}_{2} \mathrm{MnSi}$ compound, and becomes almost independent of it as soon as a low Ir doping is present (the temperature dependence is very low and almost the same for all the compositions). From these results, we can also expect that the variations of $\alpha$ are also mostly ruled by alloy disorder in the partly disordered phases of $\mathrm{Co}_{2} \mathrm{MnSi}$ that we have studied.

Several contributions to the Gilbert damping are neglected in our calculations, like magnons-magnons scattering or magnons-phonon drag: only the spin-orbit damping is taken into account. Moreover, non-quasi-particle peak effects [78] should also modify the DOS at the Fermi level which, in turn, should change the value of the Gilbert damping parameter. In addition, the bulk disordered crystals that we considered are homogeneous, whereas in real samples different kinds of disorders are often present in grains.

All the processes that we cannot consider generally increase $\alpha$ in a way that is not necessarily predictable. In addition to the different approximations made in the calculation of $\alpha$ and the contributions which are not taken into account, it is also important to keep in mind the approximations which are directly related to the DFT itself, for the calculation of the exchange-correlation energy for example. In this paper, we performed our calculations with the LSDA (see Appendix A), while, as discussed in Sec. III, previous calculations reported in the literature with the GGA, lead to a larger band gap $[56,58]$. In that case, the energy interval between the Fermi level and the bottom of the minority-spin conduction bands can become higher, which would reduce the chance that these bands overlap the Fermi level in the presence of B2-like disorders.

Finally, one has also to keep in mind that other structural defects, not discussed in this paper and linked to offstoichiometry structures could also be present, depending on the growth conditions, and modify the electronic structure and the damping parameter. Among these defects, we can mention the presence of atom vacancies, as well as other contributions more specific to thin films and nanoparticules, like surface, interface, or dimensionality effects. In particular, it has been demonstrated that $\mathrm{Mn}$-rich $\mathrm{Co}_{2} \mathrm{MnSi}$ compounds have a more robust half-metallicity, which can improve the magnetoresistance ratio of $\mathrm{Co}_{2} \mathrm{MnSi}$-based magnetic tunnel junctions [28,46,47,79-81].

\section{CONCLUSIONS}

According to our LSDA calculations, atomic disorder is a critical parameter which must be controled during the growth of $\mathrm{Co}_{2} \mathrm{MnSi}$ samples, in order to avoid the loss of the ideal electronic and magnetic properties making this material very interesting for spintronic applications. The two kinds of partial disorders which are the most likely to be found are due to $\mathrm{Mn} / \mathrm{Si}$ and $\mathrm{Co} / \mathrm{Mn}$ swaps, leading to the so-called B2-like and $\mathrm{DO}_{3}$-like phases. While the first of these phases does not change significantly the electronic and magnetic properties of $\mathrm{Co}_{2} \mathrm{MnSi}$, it makes this compound more sensitive to any additional perturbation, like a small modification of the lattice parameter. Disorders involving Co-Mn swaps ( $\mathrm{DO}_{3}$ or A2-like partial disorder) are more important as they cause more substantial changes in the electronic structure and on the magnetic properties. By decreasing drastically the magnetization and creating new electron states which will destroy the half-metallicity, such disorders are indeed responsible for a large increase of the Gilbert damping parameter $\alpha$ and can really worsen the desired performance of $\mathrm{Co}_{2} \mathrm{MnSi}$.

We gave in this paper different sets of data (spinpolarization, magnetization, spin and orbital magnetic moments or damping parameter) as a function of the kind of disorder and disorder rate, which can be used by experimentalists for future measurements in order to improve the characterization of their samples, which are never perfectly ordered but always present a partially disordered structure. Special care must be taken during the growth of $\mathrm{Co}_{2} \mathrm{MnSi}$ samples to control the alloy disorder, and to avoid the other kinds of structural defects which can modify the electronic and magnetic properties of this alloy in an undesirable way.

In addition to their interesting low damping parameter, Heusler alloys are often studied due to their half-metallicity, resulting in a high spin polarization, which is very interesting to get spintronic devices with a very high magnetoresistance. Understanding the effect of the chemical disorder on the electronic states, is thus of great interest for the spintronic community.

\section{ACKNOWLEDGMENTS}

This work was granted access to the HPC resources of CALMIP supercomputing center under the allocation p1252 (2014-2016). This project has been supported by the french Agence Nationale pour la Recherche (ANR NASSICS 12-JS10-00801). This work was supported by Programme Investissements d'Avenir under the program ANR-11-IDEX0002-02, reference ANR-10-LABX-0037-NEXT.

\section{APPENDIX A: IMPACT OF THE EXCHANGE CORRELATION FUNCTIONAL AND OF THE LLOYD FORMULA ON THE MAGNETIC PROPERTIES OF Co $\mathrm{Co}_{2} \mathrm{MnSi}$}

In this section, we study the impact of the exchangecorrelation functional and of the Lloyd formula on the magnetic moments, the DOS at the Fermi level and the Gilbert damping parameter $\alpha$ calculated for the L2 1 phase of $\mathrm{Co}_{2} \mathrm{MnSi}$. In addition to the Vosko-Wilk-Nusair (VWN) LSDA functional used throughout this paper, we performed some calculations with the Perdew-Burke-Ernzerhof (PBE) GGA functional. The experimental lattice parameter was used for all these calculations and $\alpha$ is computed, as in Sec. III, for a nearly ideal $\mathrm{L} 21$ phase with $1 \%$ of Si/Mn exchange (B2-like disorder, with $x=0.01)$. The results of the calculations have been summarized in Table III. In this table, only the majority spin density of states at the Fermi level is given, the minority spin DOS at Fermi level being equal to zero.

The atomic spin and orbital magnetic moments of the transition metals are extremely similar, whatever the functional used for the calculation. The Gilbert damping parameters obtained with both calculations are also of the same order of magnitude. The main difference between the LSDA and the GGA calculations is the magnitude of the spin magnetic moment of $\mathrm{Si}$ atoms, which is multiplied by 1.4 between the LSDA and the GGA. However, this contribution remains negligible in comparison with those of the Co and Mn atoms. 
TABLE III. Spin and orbital magnetic moments $\left(M_{\text {spin }}\right.$ and $\left.M_{\text {orb }}\right)$ for each chemical species, Gilbert damping parameter $\alpha$ and majority-spin DOS at the Fermi level $\mathrm{n}_{\uparrow}\left(E_{F}\right)$ for the perfectly ordered $\mathrm{L} 22_{1}$ phase of $\mathrm{Co}_{2} \mathrm{MnSi}$, calculated in LSDA, GGA, with and without the Lloyd formula. The Gilbert damping parameters reported in the table have been calculated for a nearly ideal L2 1 phase (B2-like disorder with $x=0.01$ ).

\begin{tabular}{|c|c|c|c|c|c|c|c|c|}
\hline & \multicolumn{2}{|c|}{$\mathrm{L} 2{ }_{1} \mathrm{GGA}$} & \multicolumn{2}{|c|}{$\mathrm{L} 2{ }_{1}$ GGA + Lloyd } & \multicolumn{2}{|c|}{$\mathrm{L} 2_{1} \mathrm{LSDA}$} & \multicolumn{2}{|c|}{$\mathrm{L} 2_{1} \mathrm{LSDA}+\mathrm{Lloyc}$} \\
\hline & $M_{\text {spin }}$ & $M_{\text {orb }}$ & $M_{\text {spin }}$ & $M_{\text {orb }}$ & $M_{\text {spin }}$ & $M_{\text {orb }}$ & $M_{\text {spin }}$ & $M_{\text {orb }}$ \\
\hline $\operatorname{Co}\left(\mu_{B} /\right.$ at $)$ & 0.94 & 0.03 & 0.98 & 0.03 & 0.97 & 0.03 & 1.01 & 0.03 \\
\hline $\operatorname{Mn}\left(\mu_{B} /\right.$ at $)$ & 3.12 & 0.02 & 3.14 & 0.02 & 3.01 & 0.02 & 3.03 & 0.02 \\
\hline $\operatorname{Si}\left(\mu_{B} /\right.$ at $)$ & -0.11 & 0.00 & -0.10 & 0.00 & -0.08 & 0.00 & -0.07 & 0.00 \\
\hline $\operatorname{Tot}\left(\mu_{B} /\right.$ f.u. $)$ & 4.88 & 0.07 & 4.99 & 0.07 & 4.87 & 0.07 & 4.99 & 0.08 \\
\hline$\alpha$ & \multicolumn{2}{|c|}{$2.02 \times 10^{-4}$} & \multicolumn{2}{|c|}{$1.80 \times 10^{-4}$} & \multicolumn{2}{|c|}{$1.54 \times 10^{-4}$} & \multicolumn{2}{|c|}{$1.65 \times 10^{-4}$} \\
\hline $\mathrm{n}_{\uparrow}\left(E_{F}\right)$ (states per eV and per f.u.) & \multicolumn{2}{|c|}{1.32} & \multicolumn{2}{|c|}{1.22} & \multicolumn{2}{|c|}{1.40} & \multicolumn{2}{|c|}{1.40} \\
\hline
\end{tabular}

The majority-spin DOS at the Fermi level only increases from about 1.3 to 1.4 (states per $\mathrm{eV}$ and per f.u.), when we switch from the LSDA to the GGA. The static and dynamic magnetic properties are here only slightly influenced by the choice of the functional. These results are in agreement with those presented in Ref. [40].

We can see, in Table III that the spin magnetic moment of the alloy is not an integer, contrary to what might be expected for a half-metallic compound. As explained by Galanakis et. al. in Ref. [57], this problem is due to an intrinsic characteristic of the KKR-Green-function formalism: to compute the Green function, the code proceeds to an angular momentum expansion of the wave function. For numerical reasons, the expansion is truncated at an integer limit value of the angular momentum $l_{\max }$ (here, $l_{\max }=4$ ). To have an integer spin magnetic moment, we should therefore impose $l_{\max } \rightarrow \infty$, which is technically impossible. This problem is often solved by the use of the Lloyd formula, which corrects the total charge and magnetic moment by an implicit summation. We therefore decided to study the impact of the Lloyd formula on the magnetic properties of the L 21 phase, calculated with the 2 functionals. The results are summarized in Table III.

The atomic magnetic moments are only slightly modified by the use of the Lloyd formula, and there is only a small effect on the Gilbert damping parameter for both functionals. With the LSDA functional, the use of the Lloyd formula does not affect the DOS at the Fermi level for the majority spin. For the GGA functional, on the other hand, we can see a decrease of $7 \%$ of this quantity when the Lloyd formula is used. The largest variation of the magnetic moments is the increase of $4.4 \%$ of the Co spin magnetic moment when we use the Lloyd formula with the GGA functional (4,2\% with the LSDA functional). The Lloyd formula corrects the total spin magnetic moment which nearly takes an integer value, as expected for a half-metallic compound. In theory, all calculations should have been done with this formula. However, given the limited impact of Lloyd formula on the Gilbert damping parameter of $\mathrm{Co}_{2} \mathrm{MnSi}$ when $l_{\max }=4$ is used, we have decided to consider these small deviations as negligible in order to avoid costly calculations.

A volume optimization of the elementary cell was performed in GGA for the four extreme phases. We found that $\mathrm{a}_{0}^{\mathrm{GGA}}\left(\mathrm{L} 2_{1}\right)=5.59 \AA$ and the lattice parameters of the totally disordered phases have, once again, differences of less than $1 \%$ with respect to the ordered one. This last series of calculations corroborates our hypothesis, which postulates that the chemical disorder has almost no impact on the lattice parameter, and confirms our choice to keep the same lattice parameter for all the considered chemical disorder rates.

It is well known that the results of DFT-based calculations vary quantitatively (and sometimes qualitatively) according to the approximation chosen for the exchange and correlation functional. However, the similarity of the results obtained with different functionals for the $\mathrm{L} 2{ }_{1}$ phase in this appendix suggests that our study would have qualitatively the same results, for a given lattice parameter, with the GGA.

\section{APPENDIX B: EXPERIMENTAL MEASUREMENTS}

We performed ferromagnetic resonance (FMR) measurements on a $\mathrm{Co}_{2} \mathrm{MnSi}$ sample irradiated with light $\mathrm{He}^{+}$ions. This technique allows to induce $\mathrm{Co} / \mathrm{Mn}$ swaps in the $\mathrm{L} 2{ }_{1}$ structure of $\mathrm{Co}_{2} \mathrm{MnSi}$; based on $\mathrm{x}$-ray diffraction in normal and anomalous conditions, along with a statistical analysis of STEM amplitudes, the $\mathrm{DO}_{3}$-like disorder rate was estimated at $z \approx 0.09$ in this sample. All the experimental details are presented in Refs. [54,75]. From the linear dependence of the linewidth with the resonance frequency shown in Fig. 9, we can extract the value of the Gilbert damping coefficient $\alpha$.

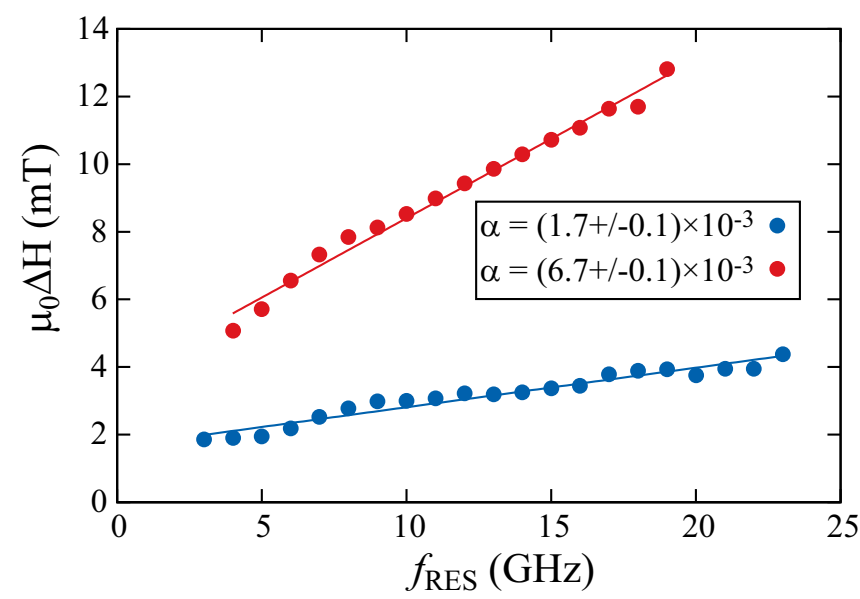

FIG. 9. FMR linewidth of a $\mathrm{Co}_{2} \mathrm{MnSi}$ irradiated sample. Blue curve: $\mathrm{L} 2{ }_{1}$ order and red curve: $\mathrm{L} 2{ }_{1}$ including $\mathrm{Mn} / \mathrm{Co}$ swaps $(x=$ $0.09)$. 
Indeed, we have $\Delta H=(2 \alpha / \gamma) f_{\mathrm{RES}}+\Delta H_{0}$, where $\gamma$ is the gyromagnetic ratio and $\Delta H_{0}$ is the extrinsic contribution to the linewidth due to magnetic and structural inhomogeneities. Separating intrinsic from extrinsic contributions is a complicated task. The details on the magnetic measurements related to this task are presented in Ref. [75] and a more general discussion on this experimental subject can be found in Ref. [82]. We observe that the damping coefficient is higher in the irradiated sample $\left(6.7 \times 10^{-3}\right)$ than in the $\mathrm{L} 2{ }_{1}$ phase $\left(1.7 \times 10^{-3}\right)$.
[1] T. L. Gilbert, IEEE Trans. Magn. 40, 3443 (2004).

[2] J. C. Slonczewski, J. Magn. Magn. Mater. 159, L1 (1996).

[3] F. Heusler, W. Stark, and E. Haupt, Verh. Dtsch. Phys. Ges. 5, 219 (1903).

[4] R. Farshchi and M. Ramsteiner, J. Appl. Phys. 113, 191101 (2013).

[5] Y. Sakuraba, M. Hattori, M. Oogane, Y. Ando, H. Kato, A. Sakuma, T. Miyazaki, and H. Kubota, Appl. Phys. Lett. 88, 192508 (2006).

[6] T. Ishikawa, T. Marukame, H. Kijima, K.-I. Matsuda, T. Uemura, M. Arita, and M. Yamamoto, Appl. Phys. Lett. 89, 192505 (2006).

[7] Y. Miura, H. Uchida, Y. Oba, K. Nagao, and M. Shirai, J. Phys.: Condens. Matter 19, 365228 (2007).

[8] Y. K. Takahashi, S. Kasai, S. Hirayama, S. Mitani, and K. Hono, Appl. Phys. Lett. 100, 052405 (2012).

[9] H. B. Huang, X. Q. Ma, Z. H. Liu, and L. Q. Chen, J. Alloys Compd. 597, 230 (2014).

[10] T. Graf, C. Felser, and S. S. P. Parkin, Prog. Solid State Chem. 39, 1 (2011).

[11] C. Felser, L. Wollmann, S. Chadov, G. H. Fecher, and S. S. P. Parkin, APL Materials 3, 041518 (2015).

[12] C. Felser and G. H. Fecher, Spintronics - From Materials to Devices (Springer, Mainz, 2013).

[13] C. Felser and A. Hirohata, Heusler Alloys - Properties, Growth, Applications (Springer, Dresden/York, 2016).

[14] S. Fujii, S. Sugimura, Ishida, and S. Asano, J. Phys.: Condens. Matter 2, 8583 (1990).

[15] R. Yilgin, M. Oogane, Y. Ando, and T. Miyazaki, J. Magn. Magn. Mater. 310, 2322 (2007).

[16] R. Yilgin, Y. Sakuraba, M. Oogane, S. Mizukami, Y. Ando, and T. Miyazaki, Jpn. J. Appl. Phys. 46, L205 (2007).

[17] M. Oogane, T. Kubota, Y. Kota, S. Mizukami, H. Naganuma, A. Sakuma, and Y. Ando, Appl. Phys. Lett. 96, 252501 (2010).

[18] F. J. Yang and X. Q. Chen, Appl. Phys. Lett. 102, 252407 (2013).

[19] M. Oogane, T. Kubota, H. Naganuma, and Y. Ando, J. Phys. D: Appl. Phys. 48, 164012 (2015).

[20] S. Qiao, J. Zhang, R. Hao, H. Zhaong, Y. Kang, and S. Kang, in Proceeding of the 2015 IEEE International Magnetics Conference (INTERMAG) (IEEE, 2015), p. 1.

[21] S.-Z. Qiao, Q.-N. Ren, R.-R. Hao, H. Zhong, Y. Kang, S.-S. Kang, Y.-F. Qin, S.-Y. Yu, G.-B. Han, S.-S. Yan, and L.-M. Mei, Chin. Phys. Lett. 33, 047601 (2016).

[22] Q. Shi-Zhu, Z. Jie, Q. Yu-Feng, H. Run-Run, Z. Hai, Z. Da-Peng, K. Yun, K. Shi-Shou, Yu Shu-Yun, H. Guang-Bing, Y. Shi-Shen, and M. Liang-Mo, Chin. Phys. Lett. 32, 057601 (2015).

[23] S. Andrieu, A. Neggache, T. Hauet, T. Devolder, A. Hallal, M. Chshiev, A. M. Bataille, P. LeFevre, and F. Bertran, Phys. Rev. B 93, 094417 (2016).

[24] C. Liu, C. K. A. Mewes, M. Chshiev, T. Mewes, and W. H. Butler, Appl. Phys. Lett. 95, 022509 (2009).

[25] A. Sakuma, J. Phys. D: Appl. Phys. 48, 164011 (2015).
[26] P. J. Webster, J. Phys. Chem. Solids 32, 1221 (1971).

[27] M. Jourdan, J. Minár, J. Braun, A. Kronenberg, S. Chadov, B. Balke, A. Gloskovskii, M. Kolbe, H. J. Elmers, G. Schönhense, H. Ebert, C. Felser, and M. Kläui, Nat. Commun. 5, 3974 (2014).

[28] K. Moges, Y. Honda, H. X. Liu, T. Uemura, M. Yamamoto, Y. Miura, and M. Shirai, Phys. Rev. B 93, 134403 (2016).

[29] M. P. Raphael, B. Ravel, M. A. Willard, S. F. Cheng, B. N. Das, R. M. Stroud, K. M. Bussmann, J. H. Claassen, and V. G. Harris, Appl. Phys. Lett. 79, 4396 (2001).

[30] M. P. Raphael, B. Ravel, Q. Huang, M. A. Willard, S. F. Cheng, B. N. Das, R. M. Stroud, K. M. Bussmann, J. H. Claassen, and V. G. Harris, Phys. Rev. B 66, 104429 (2002).

[31] J. Schmalhorst, A. Thomas, S. Kämmerer, O. Schebaum, D. Ebke, M. D. Sacher, G. Reiss, A. Hütten, A. Turchanin, A. Gölzhäuser, and E. Arenholz, Phys. Rev. B 75, 014403 (2007).

[32] R. Fetzer, J.-P. Wüstenberg, T. Taira, T. Uemura, M. Yamamoto, M. Aeschlimann, and M. Cinchetti, Phys. Rev. B 87, 184418 (2013).

[33] B. Ravel, M. P. Raphael, V. G. Harris, and Q. Huang, Phys. Rev. B 65, 184431 (2002).

[34] S. Picozzi, A. Continenza, and A. J. Freeman, Phys. Rev. B 69 , 094423 (2004).

[35] H. Pandey, R. Prasad, and R. C. Budhani, JPS Conf. Proc. 3, 017037 (2014).

[36] V. Kamberský, Can. J. Phys. 48, 2906 (1970).

[37] M. Fähnle and D. Steiauf, Phys. Rev. B 73, 184427 (2006).

[38] K. Gilmore, Y. U. Idzerda, and M. D. Stiles, Phys. Rev. Lett. 99, 027204 (2007).

[39] V. Kamberský, Czech. J. Phys. B 26, 1366 (1976).

[40] J. Chico, S. Keshavarz, Y. Kvashnin, M. Pereiro, I. Di Marco, C. Etz, O. Eriksson, A. Bergman, and L. Bergqvist, Phys. Rev. B 93, 214439 (2016).

[41] A. Köhler, L. Wollmann, D. Ebke, S. Chadov, C. Kaiser, Z. Diao, Y. Zheng, Q. Leng, and C. Felser, Phys. Rev. B 93, 094410 (2016).

[42] H. Ebert et al., The Munich SPR-KKR package, version 6.3, http://ebert.cup.uni-muenchen.de/sprkkr.

[43] H. Ebert, D. Ködderitzsch, and J. Minár, Rep. Prog. Phys. 74, 096501 (2011).

[44] S. Chadov, G. H. Fecher, C. Felser, J. Minár, J. Braun, and H. Ebert, J. Phys. D: Appl. Phys. 42, 084002 (2009).

[45] J.-P. Wüstenberg, R. Fetzer, M. Aeschlimann, M. Cinchetti, J. Minár, J. Braun, H. Ebert, T. Ishikawa, T. Uemura, and M. Yamamoto, Phys. Rev. B 85, 064407 (2012).

[46] X. Kozina, J. Karel, S. Ouardi, S. Chadov, G. H. Fecher, C. Felser, G. Stryganyuk, B. Balke, T. Ishikawa, T. Uemura, M. Yamamoto, E. Ikenaga, S. Ueda, and K. Kobayashi, Phys. Rev. B 89, 125116 (2014).

[47] R. Fetzer, S. Ouardi, Y. Honda, H.-x. Liu, S. Chadov, B. Balke, S. Ueda, M. Suzuki, T. Uemura, M. Yamamoto, M. Aeschlimann, 
M. Cinchetti, G. H. Fecher, and C. Felser, J. Phys. D: Appl. Phys. 48, 164002 (2015).

[48] P. Soven, Phys. Rev. 156, 809 (1967).

[49] H. Ebert, S. Mankovsky, D. Ködderitzsch, and P. J. Kelly, Phys. Rev. Lett. 107, 066603 (2011).

[50] A. Brataas, Y. Tserkovnyak, and G. E. W. Bauer, Phys. Rev. Lett. 101, 037207 (2008).

[51] S. Mankovsky, D. Ködderitzsch, G. Woltersdorf, and H. Ebert, Phys. Rev. B 87, 014430 (2013).

[52] S. H. Vosko, L. Wilk, and M. Nusair, Can. J. Phys. 58, 1200 (1980).

[53] J. P. Perdew, K. Burke, and M. Ernzerhof, Phys. Rev. Lett. 77, 3865 (1996).

[54] I. Abdallah, N. Ratel-Ramond, C. Magen, B. Pecassou, R. Cours, A. Arnoult, M. Respaud, J. F. Bobo, G. BenAssayag, E. Snoeck, and N. Biziere, Mater. Res. Express 3, 046101 (2016).

[55] R. Y. Umetsu, K. Kobayashi, A. Fujita, R. Kainuma, and K. Ishida, J. Appl. Phys. 103, 07 D718 (2008).

[56] S. Picozzi, A. Continenza, and A. J. Freeman, Phys. Rev. B 66, 094421 (2002).

[57] I. Galanakis, P. H. Dederichs, and N. Papanikolaou, Phys. Rev. B 66, 174429 (2002).

[58] G. H. Fecher, D. Ebke, S. Ouardi, S. Agrestini, C.-Y. Kuo, N. Hollmann, Z. Hu, A. Gloskovskii, F. Yakhou, N. B. Brookes, and C. Felser, SPIN 04, 1440017 (2014).

[59] Y. Kurtulus, R. Dronskowski, G. D. Samolyuk, and V. P. Antropov, Phys. Rev. B 71, 014425 (2005).

[60] S. Wurmehl, G. H. Fecher, K. Kroth, F. Kronast, H. A. Dürr, Y. Takeda, Y. Saitoh, K. Kobayashi, H.-J. Lin, G. Schönhense, and C. Felser, J. Phys. D: Appl. Phys. 39, 803 (2006).

[61] I. Galanakis and E. Şaşoğlu, Appl. Phys. Lett. 98, 102514 (2011).

[62] P. Làzpita, J. M. Barandiaràn, J. Gutiérrez, J. Feuchtwanger, V. A. Chernenko, and M. L. Richard, New J. Phys. 13, 033039 (2011).

[63] C. Venkatesh, V. Srinivas, V. Rao, S. Srivastava, and P. S. Babu, J. Alloys Compd. 577, 417 (2013).

[64] A. Z. Menshikov, G. A. Takzei, Y. A. Dorofeyev, V. A. Kazantsev, A. K. Kostyshin, and I. I. Sych, Zh. Eksp. I Teor. Fiz. 89, 1269 (1985) [Sov. Phys. JETP 62, 734 (1985)].

[65] T. J. Hicks and A. R. Wildes, Aust. J. Phys. 46, 667 (1993).

[66] S. Banerjee, W. O’Brien, and B. Tonner, J. Magn. Magn. Mater. 198-199, 267 (1999).
[67] S. Stadler, D. H. Minott, D. Harley, J. P. Craig, M. Khan, I. I. Dubenko, N. Ali, K. Story, J. Dvorak, Y. U. Idzerda, D. A. Arena, and V. G. Harris, J. Appl. Phys. 97, 10 C302 (2005).

[68] N. D. Telling, P. S. Keatley, G. van der Laan, R. J. Hicken, E. Arenholz, Y. Sakuraba, M. Oogane, Y. Ando, and T. Miyazaki, Phys. Rev. B 74, 224439 (2006).

[69] B. T. Thole, P. Carra, F. Sette, and G. van der Laan, Phys. Rev. Lett. 68, 1943 (1992).

[70] M. Altarelli, Phys. Rev. B 47, 597 (1993).

[71] C. T. Chen, Y. U. Idzerda, H.-J. Lin, N. V. Smith, G. Meigs, E. Chaban, G. H. Ho, E. Pellegrin, and F. Sette, Phys. Rev. Lett. 75, 152 (1995).

[72] B. Rameev, F. Yildiz, S. Kazan, B. Aktas, A. Gupta, L. R. Tagirov, D. Rata, D. Buergler, P. Gruenberg, C. M. Schneider, S. Kämmerer, G. Reiss, and A. Hütten, Physica Status Solidi (a) 203, 1503 (2006).

[73] J. Hamrle, O. Gaier, S.-G. Min, B. Hillebrands, Y. Sakuraba, and Y. Ando, J. Phys. D: Appl. Phys. 42, 084005 (2009).

[74] M. Belmeguenai, F. Zighem, D. Faurie, H. Tuzcuoglu, S.-M. Chérif, P. Moch, S. W. Westerholt, and W. Seiler, Physica Status Solidi 209, 1328 (2012).

[75] I. Abdallah, B. Pradines, N. Ratel-Ramond, G. BenAssayag, R. Arras, L. Calmels, J. F. Bobo, E. Snoeck, and N. Biziere, J. Phys. D: Appl. Phys. 50, 035003 (2017).

[76] A. A. Starikov, P. J. Kelly, A. Brataas, Y. Tserkovnyak, and G. E. W. Bauer, Phys. Rev. Lett. 105, 236601 (2010).

[77] H. Ebert, S. Mankovsky, K. Chadova, S. Polesya, J. Minár, and D. Ködderitzsch, Phys. Rev. B 91, 165132 (2015).

[78] L. Chioncel, Y. Sakuraba, E. Arrigoni, M. I. Katsnelson, M. Oogane, Y. Ando, T. Miyazaki, E. Burzo, and A. I. Lichtenstein, Phys. Rev. Lett. 100, 086402 (2008).

[79] K. Özdoğan, E. Şaşoğlu, and I. Galanakis, physica status solidi (RRL) - Rapid Research Letters 1, 184 (2007).

[80] M. Yamamoto, T. Ishikawa, T. Taira, G.-f. Li, K.-i. Matsuda, and T. Uemura, J. Phys.: Condens. Matter 22, 164212 (2010).

[81] B. Hu, K. Moges, Y. Honda, H.-x. Liu, T. Uemura, M. Yamamoto, J.-i. Inoue, and M. Shirai, Phys. Rev. B 94, 094428 (2016).

[82] M. A. W. Schoen, D. Thonig, M. L. Schneider, T. J. Silva, H. T. Nembach, O. Eriksson, O. Karis, and J. M. Shaw, Nat. Phys. 12, 839 (2016). 